\title{
Clinicopathological analysis of HOXD4 expression in diffuse gliomas and its correlation with IDH mutations and 1p/19q co- deletion
}

\author{
Xin-Wei Zhao ${ }^{1, *}$, Yun-Bo Zhan ${ }^{1, *}$, Jian-Ji Bao ${ }^{1}$, Jin-Qiao Zhou ${ }^{1}$, Feng-Jiang Zhang ${ }^{1}$, \\ Yu Bin ${ }^{1}$, Ya-Hui Bai ${ }^{1}$, Yan-Min Wang ${ }^{1}$, Zhen-Yu Zhang ${ }^{1}$ and Xian-Zhi Liu ${ }^{1}$ \\ ${ }^{1}$ Department of Neurosurgery, The First Affiliated Hospital of Zhengzhou University, Zhengzhou, Henan 450001, China \\ *These authors have contributed equally to this work \\ Correspondence to: Zhen-Yu Zhang, email: neurozzy@foxmail.com \\ Xian-Zhi Liu, email: xzliv06@126.com \\ Keywords: gliomas; HOXD4; immunohistochemistry; TCGA; prognosis \\ Received: November 03, $2017 \quad$ Accepted: December 05, $2017 \quad$ Published: December 18, 2017 \\ Copyright: Zhao et al. This is an open-access article distributed under the terms of the Creative Commons Attribution License 3.0 \\ (CC BY 3.0), which permits unrestricted use, distribution, and reproduction in any medium, provided the original author and source \\ are credited.
}

\section{ABSTRACT}

Backgrounds: HOX (homologous box) is known as the dominant gene of vertebrate growth and cell differentiation. Abnormal expression of HOX gene in various tumors has attracted the attention of scholars. As a component of HOX clusters, HOXD4 plays a controversial role in the tumorigenesis of central nervous system.

Results: The data demonstrated that and the results demonstrated that HOXD4 was overexpressed in glioma tissues compared to that of normal brain tissues. patients with high HOXD4 expression had a significant shorter survival than those with low HOXD4 expression in total glioma cohort $(p<0.001)$, WHO Grade II cohort $(p=0.003)$ and Grade III cohort $(p<0.001)$, but not in Grade IV cohort when OS (overall survival) was analyzed $(p=0.216)$. The findings were confirmed by the large-scale omics data analysis including lower-grade glioma (LGG) and glioblastoma multiforme (GBM) in TCGA (the cancer genome atlas) and CGGA (Chinese glioma genome atlas). Moreover, it was revealed that the expression of HOXD4 have a significant impact on the OS of Grade IV glioma with IDH wild-type and 1p/19q intact according to TCGA data.

Methods: Clinicopathological analysis of HOXD4 expression in 453 glioma patients was performed in the current study. Expression of HOXD4 was evaluated by qPCR and immunohistochemical (IHC) staining. Univariate and multivariate analysis were conducted to investigate the prognostic role of HOXD4 in glioma patients.

Conclusions: Expression of HOXD4 was closely related to the clinical outcomes of patients with gliomas, and HOXD4 may be a potential prognostic biomarker of gliomas.

\section{INTRODUCTION}

Glioma is recognized as the most common malignant neoplasm occurred in the central nervous system [1]. Gliomas ranked the first in the incidence of intracranial tumors, and were characterized by high aggressiveness, recurrence rate and mortality [2]. According to the WHO classification, gliomas are divided into four degrees [3]. There are many hypotheses about the pathogenesis of glioma, such as genetic factors, radiation exposure and nitrite contact [4]. At present, the treatments of gliomas include surgical resection with postoperative comprehensive treatment (radiotherapy, chemotherapy and gene targeting therapy). Nevertheless, the survival times of glioma patients are not significantly prolonged, especially in glioblastoma patients, whose median survival time is only 12-14 months [5-11]. Due to highly invasive nature of gliomas, postoperative treatments cannot prevent tumor 
recurrence [12]. Meanwhile, side effects of radiotherapy and chemotherapy on patients make more harm than good [13]. To stratify and improve the prognosis of glioma patients, it is increasingly important to identify more potential biomarkers and therapeutic targets.

HOX gene, which is also known as homologous box gene, has a close relationship with the early embryo development [14-16]. As the main gene of vertebrate growth and cell differentiation, it plays an important role in the development of central nervous system, axial bone, gastrointestinal tract, incontinence, external genitalia and limb $[17,18]$. A total of 39 homologous box sequences were found in the mice and humans, which could be divided into 13 groups according to the position on the chromosome [19]. In the previous study, the HOX gene was overexpressed by investigating the spontaneously derived tumor-bearing canine breast cancer model. The expression profile was consistent with the oncogenelike features (HOXA1, HOXA13, HOXD4, HOXD9 and SIX1) [20]. It was also reported that HOX cluster genes promoted the proliferation and differentiation of neuroblastoma cells [21]. But the impact of HOXD cluster genes in glioma has not been clarified up to now.

In the current study, we identified the up-regulated expression of HOXD4 in diffuse glioma tissues. Meanwhile, expression of HOXD4 was revealed an independent prognostic factor in patients with gliomas. Furthermore, data acquired from TCGA and CGGA were also analyzed to corroborate our findings.

\section{RESULTS}

\section{Expression of HOXD4 was elevated in gliomas and public database (TCGA and CGGA)}

To investigate the expression of HOXD4, qPCR was performed in a panel of 44 glioma tissues including 23 primary GBMs (Grade IV), 10 AAs (Grade III) and 11 OAs (Grade II), as well as 10 non-neoplastic brain tissues for control. As results was shown (Figure 1B), One-way Anova analysis with Bonferroni correction demonstrated that the expression of HOXD4 was remarkably elevated in gliomas than normal brain $(p=0.047)$. Meanwhile, the expression of HOXD4 is significantly higher in GBM than LGG as well ( $p=0.004)$.

To further confirm our revealings, RNA sequences of TCGA and CGGA were acquired and analyzed to identify the HOXD4 expression and its prognostic role in gliomas. Bioconductor/TCGA biolinks function package from TCGA (https://tcga-data.nci.nih.gov/tcga/) was used to download and pretreat GBM and LGG mRNA expression RNASEqV2 data. Expression-logs of HOXD4 of gliomas in mRNA level was analyzed by One-way Anova method, and results demonstrated that the expression of HOXD4 was significantly higher in WHO grade IV gliomas than Grade IIIgliomas ( $p<0.001$ Figure 1C). The expression of
HOXD4 was considerably higher in WHO grade III gliomas than Grade II gliomas $(p<0.001)$. Similarly, we conducted One-way Anova analysis to analyze the CGGA data. The expression of HOXD4 in glioma tissues was ladder-like elevated as the pathological grades escalated (Figure 1D), which is in accordance with the results of TCGA data analysis. The staining was localized mainly in the nucleus, with a small amount of cytoplasm. High expression of HOXD4 was determined in 178 samples, consisting of $39.29 \%$ in all glioma samples while the others were considered as low expression (60.71\%). We calculated the average values of the staining scores of HOXD4 expression in normal brain tissues and gliomas. The staining scores corresponding to brain tissue and Grade II, III, IV gliomas was $0.5 \pm 0.14,2.1 \pm 0.55,3.3 \pm 0.63,4.8 \pm 0.59$ respectively (Figure 2A-2D). One-way Anova analysis with Bonferroni correction revealed that staining score of HOXD4 was higher in glioma than normal brain $(p=0.021)$, and also significantly higher in GBM than LGG $(p=0.001)$.

\section{HOXD4 was revealed as an independent prognostic factor in glioma patients.}

A total of 527 patients were followed up from 2011 to 2014 at the First Affiliated Hospital of Zhengzhou University, and clinical data of 453 patients were collected, missing rate was $14.1 \%$, the median durational follow-up time was 25.9 months. The deadline of follow-up was 2017.8.30. Kaplan-Meier survival curve was used to demonstrate the relationship between HOXD4 expression and survival times of glioma patients. Results showed that in total cohort, patients with high HOXD4 expression have a significantly worse OS and PFS than those with low HOXD4 expression (Figure 2E $p<0.001$ ). Meanwhile, in different grades of gliomas, univariate analysis demonstrated that patients with high HOXD4 expression had a considerably shorter OS than that with low HOXD4 expression in Grade II (Figure $2 \mathrm{~F} p=0.003$ ) and Grade III glioma cohorts (Figure 2G $p<0.001$ ), but not in Grade IV gliomas (Figure $2 \mathrm{H} p=0.216$ ). The clinicopathological features of the glioma cohort was summarized according to the HOXD4 expression level (Table 1). HOXD4 IHC scores was high in 219 samples and low in 234 samples according to the cut-off score by ROC curve. There was significant association between HOXD4 IHC score and patient age, recurrence, or WHO Grades ( $p>$ 0.05 for all covariates). Univariate analysis demonstrated that patients age $(p<0.001)$, extent of resection $(\mathrm{p}=0.012)$, postoperative radiation therapy $(p<0.001)$, postoperative chemotherapy $(p<0.001)$ and HOXD4 expression $(p<0.001)$ impact the prognosis in total glioma patients. In multivariate survival analysis, we included prognostic factors with significance such as complete resection, radiotherapy, chemotherapy, Karnofsky Performance Status (KPS score) and expression of HOXD4. Data analysis demonstrated that HOXD4 expression was an independent prognostic factor significantly influencing the survival of patients with gliomas (Table 2). 


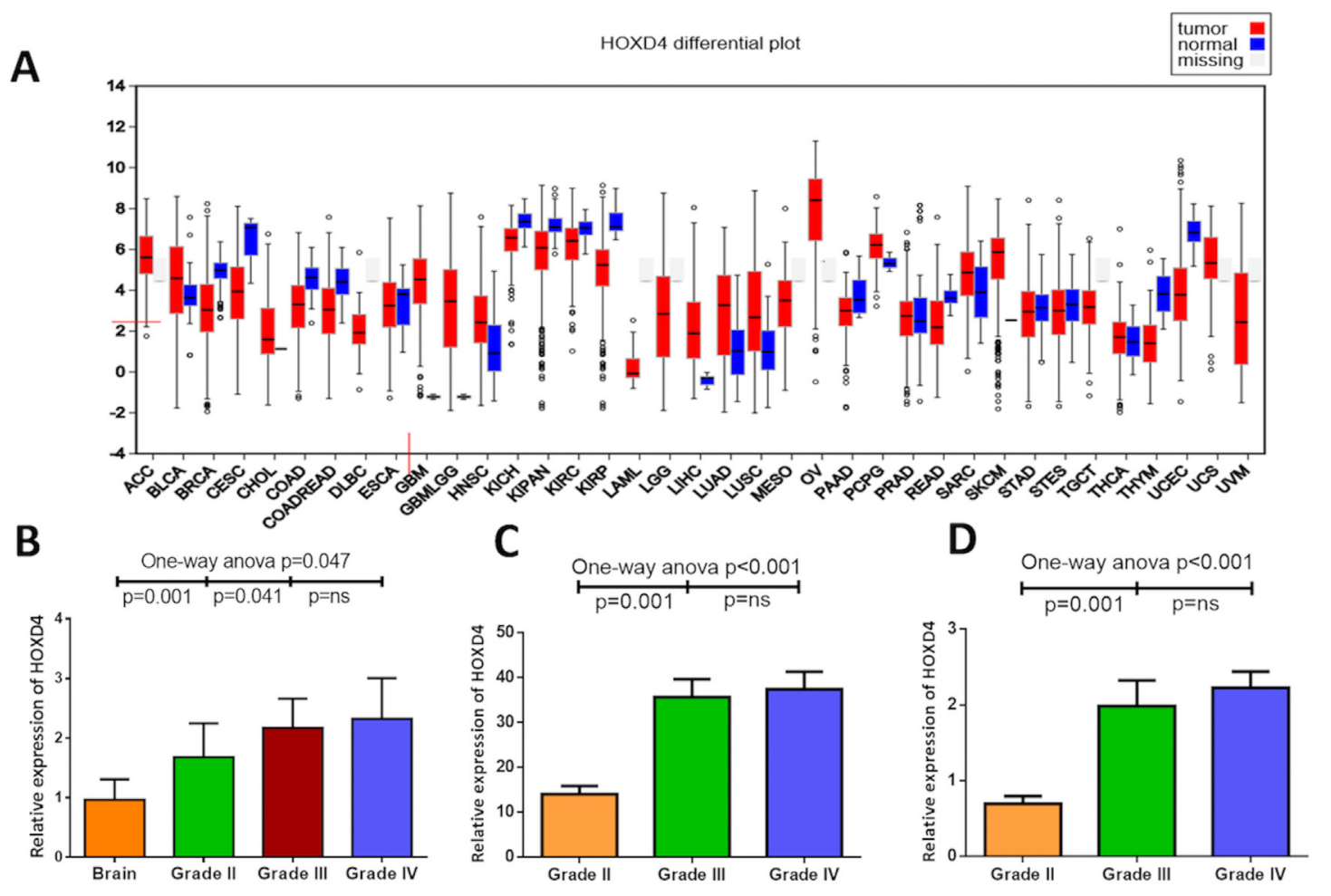

Figure 1: Abnormal expression of HOXD4 in diffuse glioma at mRNA level. (A) HOXD4 expression at FireBrowse (http:// firebrowse.org/viewGene.html?gene=HOXD4). (B) Relative quantification (RQ) values of HOXD4 by PCR showed a higher HOXD4 expression in Grade II glioma than brain $(p=0.001)$, and higher in Grade III compared with II $(p=0.041)$ while not significant between Grade III and IV ( $p=$ ns). (C) Reads per kilobase per million (RPKM) of HOXD4 from TCGA was calculated $(p=0.001)$, significantly higher in Grade III glioma than II ( $p=0.001)$, not in Grade IV than III ( $p=$ ns). (D) $\log _{2}(\mathrm{RPKM}+1)$ of HOXD4 was calculated from CGGA ( $p=0.001)$, significantly higher in Grade III glioma than II $(p=0.001)$, not in Grade IV than III ( $p=n s)$.

A

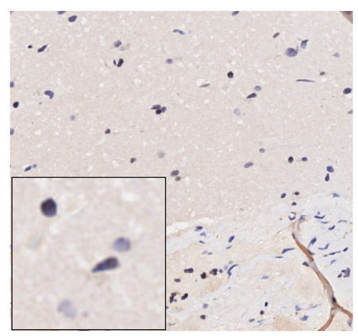

C

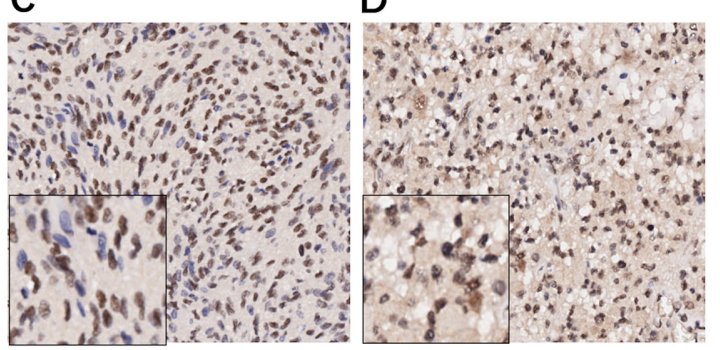

B

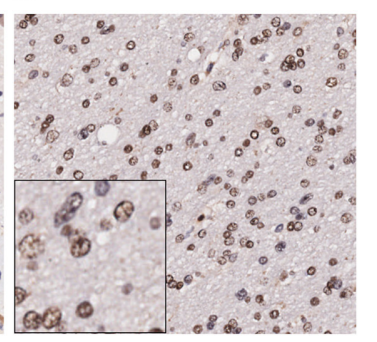

D
$E$

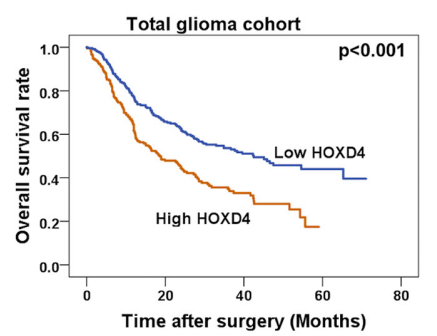

G

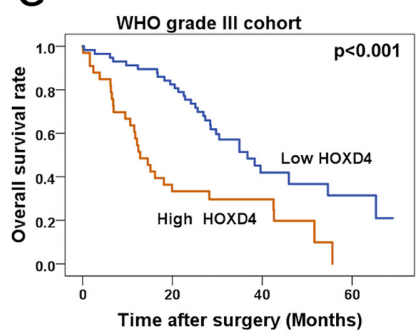

$\mathrm{F}$

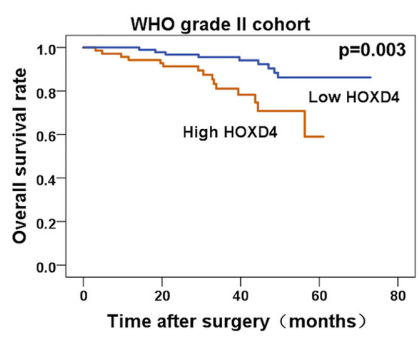

$\mathrm{H}$

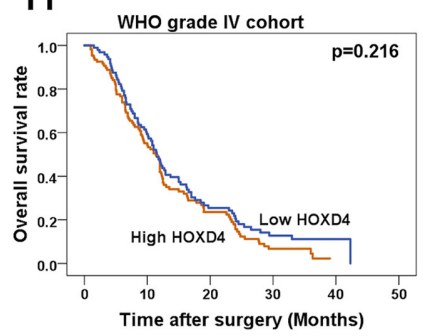

Figure 2: Immunohistochemical staining of HOXD4 in glioma and survival analysis of 453 patients by Kaplan-Meier method. (A-D) FFPE tissues with HOXD4 expression of normal brain, gliomas of WHO grade II, III and IV respectively ( $\times 200$, scale bars $50 \mu \mathrm{m})$, the left bottom of the picture is the enlarged version $(\times 100)$. (E-H) Overall survival curve by Kaplan-Meier method in cohorts of total glioma patients $(p<0.001)$, glioma WHO grade II $(p=0.003)$, III $(p<0.001)$ and IV $(p=0.216)$ respectively. 
Table 1: Association between HOXD4 expression by IHC and clinicopathological features of 453 glioma patients

\begin{tabular}{|c|c|c|c|c|}
\hline Factors & No. of cases & High HOXD4 $n=219$ & Low HOXD4 n =234 & P-value \\
\hline \multicolumn{5}{|l|}{ Sex } \\
\hline Male & 256 & 123 & 133 & 0.885 \\
\hline Female & 197 & 96 & 101 & \\
\hline \multicolumn{5}{|l|}{ Age } \\
\hline$\leq 40$ & 67 & 35 & 32 & 0.010 \\
\hline$>40$ & 386 & 184 & 202 & \\
\hline \multicolumn{5}{|l|}{ "KPS } \\
\hline$\leq 80$ & 148 & 74 & 74 & 0.623 \\
\hline$>80$ & 305 & 145 & 160 & \\
\hline \multicolumn{5}{|l|}{ Extent of resection } \\
\hline Gross total & 343 & 150 & 193 & 0.001 \\
\hline Subtotal & 110 & 69 & 41 & \\
\hline \multicolumn{5}{|l|}{ Recurrence } \\
\hline Yes & 282 & 154 & 128 & 0.002 \\
\hline No & 171 & 65 & 106 & \\
\hline \multicolumn{5}{|l|}{ Grade } \\
\hline II & 160 & 70 & 90 & 0.013 \\
\hline III & 90 & 35 & 55 & \\
\hline IV & 203 & 113 & 90 & \\
\hline \multicolumn{5}{|l|}{ Histology } \\
\hline Astrocytoma & 85 & 35 & 50 & 0.086 \\
\hline Oligodendroglioma & 165 & 81 & 85 & \\
\hline Glioblastoma & 203 & 112 & 91 & \\
\hline
\end{tabular}

The test statistic is Fisher's exact test; two tailed P value.

\section{The expression of HOXD4 influence the prognosis of glioma patients according to the data from TCGA and CGGA}

By the matching of barcode each sample allocated by TCGA, in 595 LGG and 165 GBM patients, we mapped each patient-s clinical data (including OS) to its RNA sequence. These cases were divided into group II, III and IV according to the WHO grades as well. In each cohort, the cases were separated into high HOXD4 expression group and low HOXD4 expression group according to expression-log of HOXD4. As the KaplanMeier survival curve revealed, in total glioma patients (Figure $3 \mathrm{~A} p<0.001$ ) and WHO II (Figure 3B $p=0.001$ ), III (Figure 3C $p<0.001$ ), patients with high HOXD4 expression have a significantly shorter OS than those with low HOXD4 expression. However, HOXD4 expression is not a prognostic factor of significance in Grade IV gliomas
(Figure 3D $p=0.077$ ). The cases from CGGA included 181 LGG and 144 GBM patients with the matching RNA sequence. Univariate analysis demonstrated that group with low HOXD4 expression had a considerably better OS than group of high HOXD4 expression in total glioma patients (Figure $3 \mathrm{E} p<0.001$ ) and WHO II (Figure $3 \mathrm{~F}$ $p<0.001$ ), III (Figure $3 \mathrm{G} p=0.012$ ). There is no statistical significance between HOXD4 expression and survival time in Grade IV glioma cohort (Figure $3 \mathrm{H} p=0.100$ ).

\section{HOXD4 expression influences the OS in GBM patients with $I D H$ wild-type and $1 \mathrm{p} / 19 \mathrm{q}$ intact}

In a further investigation of TCGA data, we also seek the gene mutation and chromosome gene copy number variation of glioma cohort, and then acquired $I D H$ mutations and $1 \mathrm{p} 19 \mathrm{q}$ co-deletion in these samples. In $I D H$ wild-type subgroup of GBM, it was revealed that patients 
Table 2: Multivariate Cox Proportional-Hazards Models for gliomas

\begin{tabular}{|c|c|c|c|c|}
\hline \multirow[t]{2}{*}{ Factors } & \multicolumn{2}{|c|}{$\mathbf{O S}$} & \multicolumn{2}{|c|}{ PFS } \\
\hline & OR (95\% CI) & $p$ value & OR (95\% CI) & $p$ value \\
\hline $\mathrm{KPS}^{\mathrm{a}}(\leq 80$ or $>80)$ & $1.224(0.94-1.60)$ & 0.136 & $1.15(0.89-1.49)$ & 0.259 \\
\hline $\begin{array}{l}\text { Complete resection } \\
\text { (yes or no) }\end{array}$ & $1.290(0.97-1.71)$ & 0.075 & $1.28(0.98-1.68)$ & 0.071 \\
\hline $\mathrm{RT}^{\mathrm{b}}$ (yes or no) & $2.040(1.48-2.88)$ & 0.001 & $1.91(1.37-2.68)$ & 0.001 \\
\hline $\mathrm{CHT}^{\mathrm{c}}$ (yes or no) & $1.148(0.82-1.60)$ & 0.411 & $1.07(0.76-1.47)$ & 0.688 \\
\hline $\begin{array}{l}\text { HOXD4 expression } \\
\text { (high or low) }\end{array}$ & $0.468(0.36-0.60)$ & 0.001 & $0.53(0.42-0.68)$ & 0.001 \\
\hline
\end{tabular}

Bold values indicate $p<0.05$.

OR odds ratio, CI confidence interval.

a Indicating Karnofsky Performance Status.

${ }^{\mathrm{b}}$ Indicating postoperative primary radiation therapy.

${ }^{\mathrm{c}}$ Indicating postoperative primary chemotherapy.

with high HOXD4 expression had significantly worse OS than that of patients with low HOXD4 expression (Figure $4 \mathrm{~A} p=0.013$ ), while this phenomenon was not observed in GBM patients with $I D H$ mutation (Figure $4 \mathrm{~B} p=0.302$ ). Moreover, analysis of Copy Number Variations (CNVs) in LGG and GBM samples from TCGA was conducted by Integrative Genomics Viewer (Figure 4E, 4F). in survival analysis demonstrated that HOXD4 expression were a remarkable prognostic factor in $1 \mathrm{p} / 19 \mathrm{q}$ intact subgroup of GBM (Figure $4 \mathrm{C} p=0.001$ ), but not in $1 \mathrm{p} / 19 \mathrm{q}$ co-deletion subgroup (Figure 4D $p=0.090$ ).

\section{DISCUSSION}

In the current study, the results of PCR and immunohistochemical staining demonstrated that HOXD4 overexpression significantly correlated with the malignancy of gliomas and had a remarkable impact on the prognosis of glioma patients, and the revealing were also confirmed by statistical analysis from data of TCGA and CGGA.

HOX, short for homeo box, is named by its distinctive sequence which contains $180-183$ bps [23].
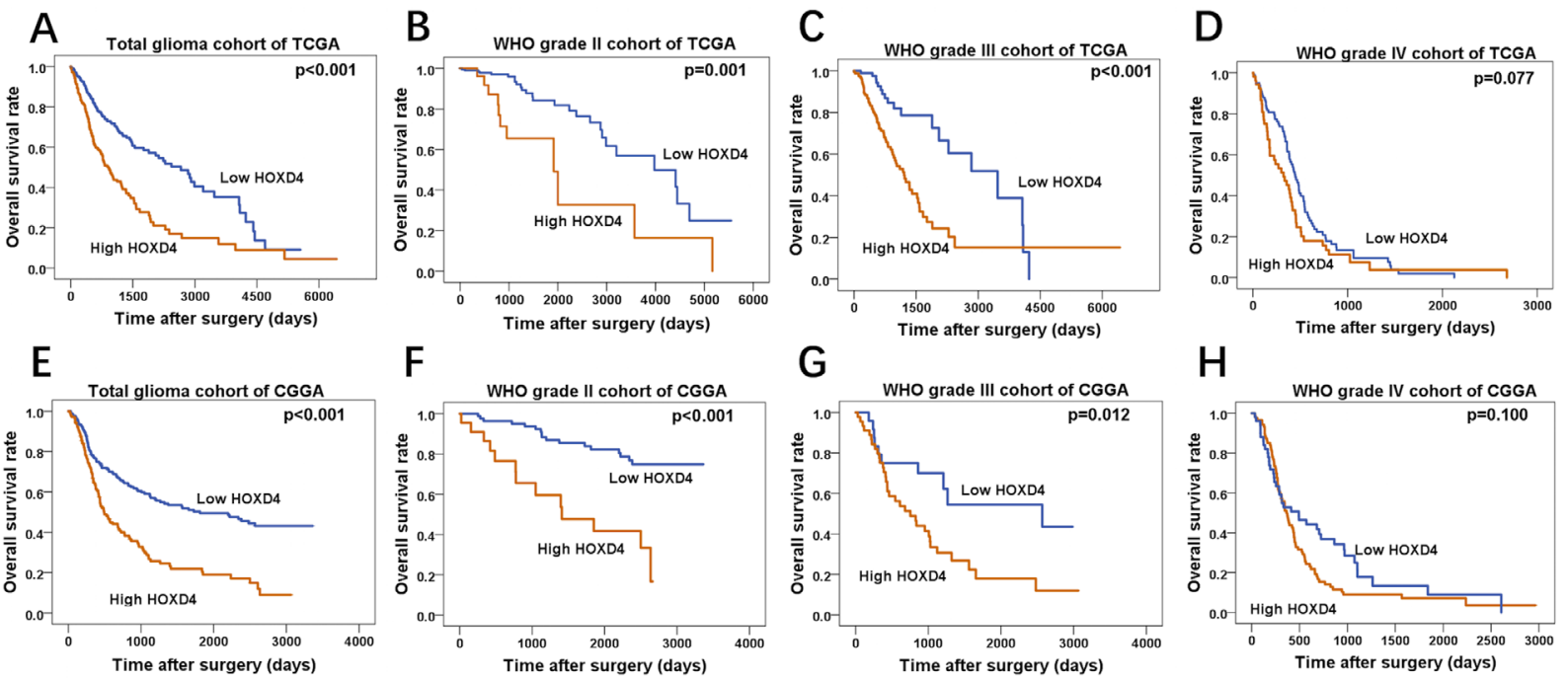

Figure 3: Survival analysis of TCGA and CGGA glioma cases. (A-D) Overall survival curve by Kaplan-Meier method for cohorts of TCGA, High HOXD4 expression cause a shorter survival period of patients in total glioma $(p<0.001)$ and WHO II ( $p=0.001)$, III $(p<0.001)$ cohorts than low HOXD4, while there is no significant correlation in WHO IV glioma $(p=0.077)$. (E-H) Overall survival curve by Kaplan-Meier method for cohorts of CGGA, patients with high HOXD4 have an unfavorable prognosis in total glioma $(p<0.001)$ and WHO II $(p<0.001)$, III $(p=0.012)$ cohorts than low HOXD4, WHO IV glioma cohorts show no difference $(p=0.100)$. 
A

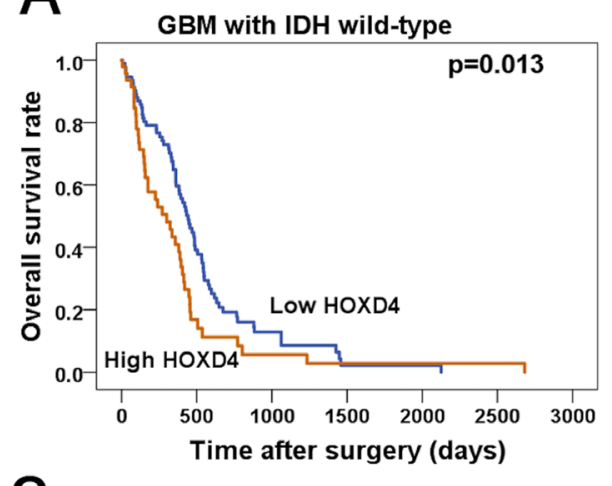

C

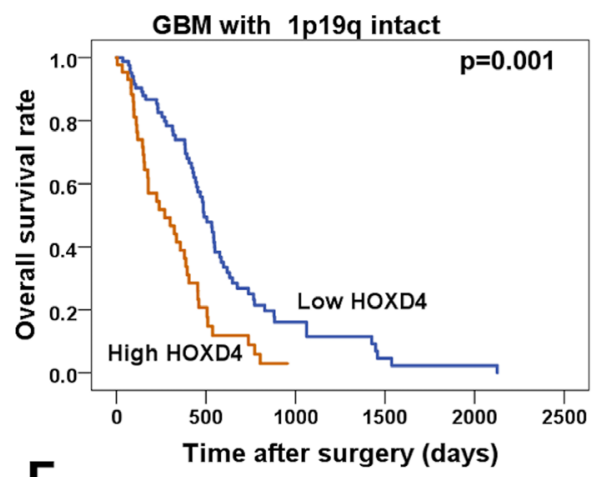

B

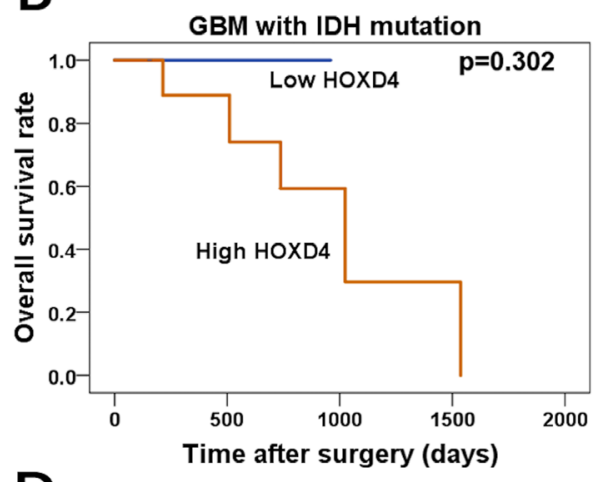

D

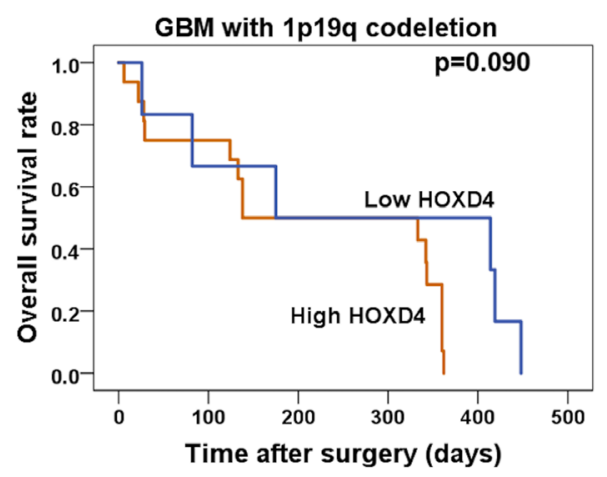

E

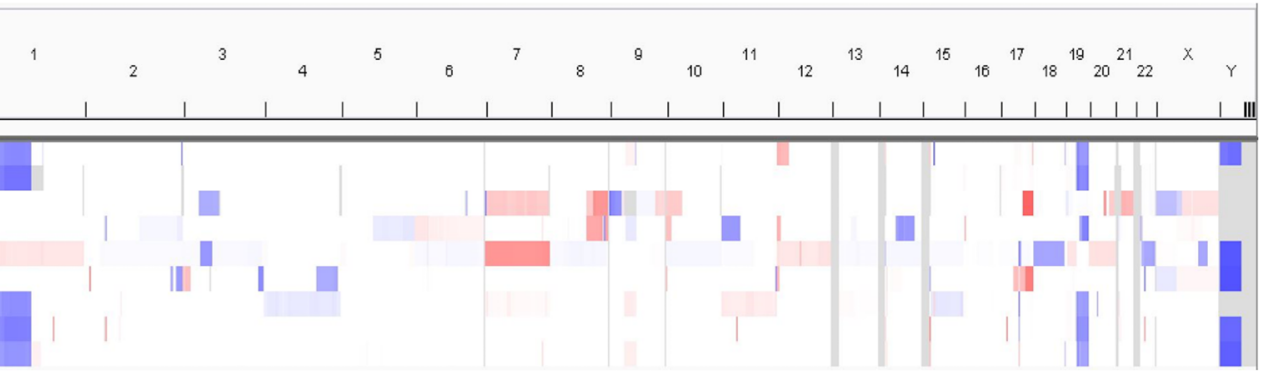

$\mathbf{F}$

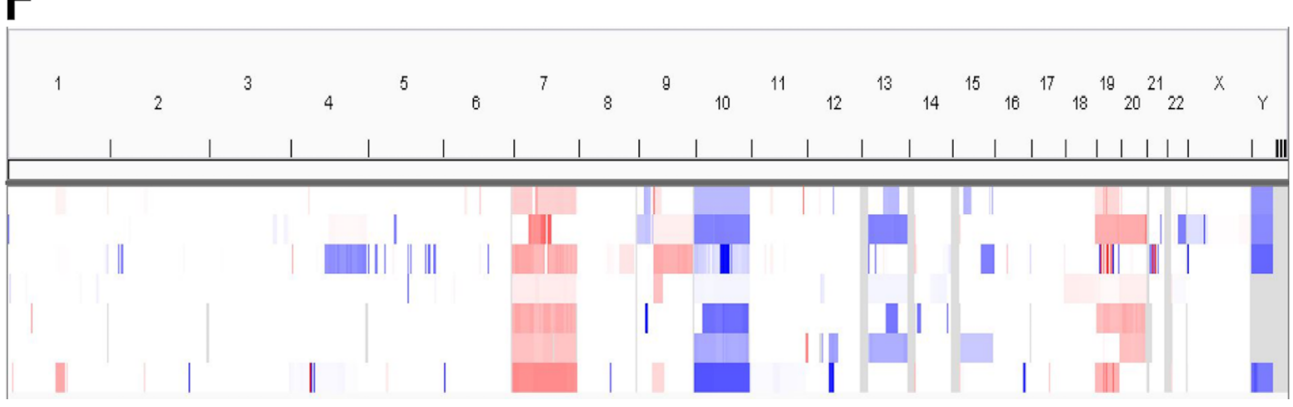

Figure 4: HOXD4 expression impact the OS of GBM patients with $I D H$ wild-type or 1p19q intact by TCGA data analysis. (A-B) Prognosis of GBM with $I D H$ wild-type was associated with HOXD4 expression $(p=0.013)$, but prognosis of GBM with $I D H$ mutation was not significantly associated with HOXD4 expression ( $p=0.302$ ). (C-D) Prognosis of GBM with $1 \mathrm{p} 19 \mathrm{q}$ intact was associated with HOXD4 expression $(p=0.001)$, but prognosis of GBM with $1 \mathrm{p} 19 \mathrm{q}$ codeletion was not significantly associated with HOXD4 expression $(p=0.090)$. (E-F)Analysis of Copy Number Variations (CNVs) in LGG and GBM samples from TCGA, respectively. Rows represent glioma patients and columns represent chromosomes. The colors in the heatmap indicate allelic states: white =normal heterozygous state; light red =imbalanced gain; Dark red =amplified heterozygous states; Light blue = copy neutral LOH and amplified LOH; Dark blue $=\mathrm{LOH}$ or physical loss in the context of a diploid genome. 
60-61 amino acids encoded by this sequence form a polypeptide region, which is known as the homeodomain. Expression of downstream target gene was regulated by the combination of homeodomain and specific DNA [14]. Studies have shown that HOX gene clusters played an important role in the progression in a variety of tumors. In the leukemia characterized by BCR/ABL and MLL fusion gene, the expression of HOX was elevated. Further studies revealed that HOX was a downstream target of MLL and HOX fusion gene, and involved in the formation of myeloid phenotype [24]. Expression of HOX clusters showed a dramatic inhibition caused by miRNA-10a in breast cancer, suggesting that they may play a functional role in the process of breast cancer [25]. In glioma development and progression, there are quite a few reports about the functions of HOX clusters. Previous studies found that HOXA9 in human gliomas promoted cell proliferation, and inhibited the apoptosis by suppressing PI3K-AKT pathway, which contributed to the development of tumors [26]. Besides, HOXA13 is a diagnostic marker for GBM and activated Wnt/ TGF- $\beta$ to promote glioma development [27]. Analysis of HOXD gene expression in human low-grade gliomas tissues revealed that HOXD1 and HOXD12 were highly expressed in gliomas, whereas the expression of HOXD3 was depressed [28]. It was also found that HOXD9 could promote the proliferation of glioma cells and inhibit cell apoptosis, the research identified the high expression of HOXD9 functioned as an enriched-cell fraction of glioma cancer stem-like cells [29]. In summary, the HOX gene is strongly associated with the pathogenesis of gliomas. and it should be emphasized that, when comparing with HOX gene research in lung cancer, leukemia and other tumors in the study, HOX gene still need deeper study for the molecular treatment of glioma [30].

In our current study, the expression of HOXD4 in Grade II, III, IV gliomas and normal brain tissue samples was detected by qRT-PCR, indicating that HOXD4 is widely present in glioma tissues. However, in the comparison of tumor tissue and normal brain tissue, we found that HOXD4 expression was significantly increased, suggesting that HOXD4 may play a role in the progression of gliomas. Multivariate survival analysis including putative prognostic factors such as KPS [31, 32], radiation therapy, chemotherapy, resection extent [33] demonstrated that HOXD4 was an independent prognosis factor in glioma patients. Furthermore, we analyzed the expression and survival data of TCGA and CGGA cohort concerning LGG and GBM on HOXD4, and revealed that HOXD4 expression was significantly increased in GBM patients compared to LGG patients. These data from the databases above further validated the preliminary results on HOXD4 derived from the data of our cohort.

In recent years, discovery of $I D H$ mutations is one of the most important findings in glioma genomics. $I D H$ mutations have a definite relationship with the survival of glioma patients, which has been widely recognized [34]. As $I D H$ has a clear impact to the prognosis of glioma patients, survival analysis of GBM patients with $I D H$ wild-type may be another solution for studying the effects of HOXD4 on clinical outcomes [35]. And $I D H$-wildtype glioblastoma could be defined clinically as primary glioblastoma [36]. Therefore, we further divided LGG and GBM cohorts of TCGA into different subgroups according to $I D H$ mutation. It was hypothesized that HOXD4 could be considered as a potential predictor of GBM prognosis. For the similar reasons, GBM patients of $1 \mathrm{p} / 19 \mathrm{q}$ intact was established as another model for the research of HOXD4 on the prognosis. And it was identified that HOXD4 was a potential predictor in GBM patients with intact $1 p / 19 q$ chromosome.

There were also several limitations in the current research. First, although the sample size of our cohort was quite large and the conclusion was corroborated by enormous scale of samples in TCGA and CGGA database, the nature of the study is retrospective. Further prospective studies were needed to confirm our findings. Second, further experiments focused on molecular mechanism of HOXD4 in glioma carcinogenesis and progression was needed in the future.

In conclusion, the current results demonstrated the expression of HOXD4 was elevated in gliomas and closely correlated with the malignancy of gliomas. Moreover, HOXD4 was revealed as a potential prognostic factor in a large cohort by univariate and multivariate analysis. HOXD4 may be an important prognostic factor and a potential therapeutic target for glioma in the future.

\section{MATERIALS AND METHODS}

\section{Patients, specimens and clinical data}

The research was approved by the Human Scientific Ethics Committee of Zhengzhou University. 453 patients received surgery between November 2011 and December 2015 in The First Affiliated Hospital of Zhengzhou University were included in the current research. All cases were stained with hematoxylin \& eosin (H\&E) and centrally reviewed according to the 2007 World Health Organization (WHO) criteria [22]. Clinical characteristics of patients were retrieved from medical documents. The follow-up data was obtained by telephone-calls or out-patient clinic. Formalin-fixed paraffin-embedded (FFPE) tissues of primary tumor were collected simultaneously. Fresh-frozen glioma tissues including 11 cases of anaplantic astrocytomas (AAs), 10 cases of anaplastic oligodendrogliomas (OAs), 23 cases of GBMs and 10 normal brain tissues (collected in brain trauma surgery) were gathered in The First Affiliated Hospital of Zhengzhou university between August 2016 and November 2016. All patients had signed informed consents before tissue collection. 


\section{TCGA and CGGA data analysis}

Information of 595 LGG cases and 165 GBM cases was collected from TCGA. The data files included: (1) RNA sequencing; (2) DNA copy-number and singlenucleotide polymorphism arrays; (3) CNV-DNA copy; (4) Clinical data of patients. OS and PFS were computed by using the Kaplan-Meier survival curves and compared by the log-rank test. The cases were separated by WHO classification and then analyzed. The cut-off scores were calculated by the X-tile software (http://medicine.yale.edu/ lab/rimm/research/software.aspx).

Data was explored through the following online data libraries:

the Broad Institute FireBrowse portal (http:// firebrowse.org/?cohort=GBMLGG),

the cBioPortal for Cancer Genomics (http://www. cbioportal.org/cancerid=lgggbm_tcga_pub), nih.gov/),

the Cancer Genome Atlas (https://cancergenome.

the Chinese Glioma Genome Atlas (http://www. cgga.org.cn/),

the TCGA publication page (https://tcga-data.nci. nih.gov/docs/publications/lgggbm_2015/).

\section{Real-time quantitative PCR}

Fresh-frozen glioma tissues was moved out from $-80^{\circ} \mathrm{C}$ and grinded into tissue suspension, Trizol (Invitrogen, Carlsbad, US) was applied for extracting the RNAs of total cells, then the cDNA reverse transcription is accomplished by the using of RT Primer Mix and PrimeScript RT Enzyme Mix 1 (Takara, Japan), reaction of reverse transcription included 50ng of RNA, 4ul of $5 \times$ PrimeScript Buffer, 2 ul of PrimeScript RT Enzyme Mix, The reaction was incubated for $15 \mathrm{~min}$ at $42^{\circ} \mathrm{C}$, qRT-PCR was made by SYBR Premix Ex Taq (Takara, Japan), QRTPCR was performed according to the SYBR ${ }^{\circledR}$ Premix Ex Taq TM II (Tli RNaseH Plus) kit instructions. Reaction conditions: $95^{\circ} \mathrm{C}$ pre-denaturation $30 \mathrm{~s} ; 95^{\circ} \mathrm{C}$ denatured $5 \mathrm{~s}, 58^{\circ} \mathrm{C}$ annealed $34 \mathrm{~s}$, For 40 cycles. GAPDH was used as an internal standard, primers sequence used as follows:

Hoxd4-F: CTAGTCGCCGGCTGCGGGAT

Hoxd4-R: TTAGTCCCCCGGAGGGTGCG

GAPDH-F: 5'- ACGGATTTGGTCGTATTGGG -3'

GAPDH-R: 5'- TCATTTTGGAGGGATCTCGC -3'

After the reaction, the SDSShell software records the number of cycles that each hole reaches the set fluorescence threshold, the $\mathrm{CT}$ value. The average data of the HOXD4 was analyzed by $\mathrm{Ct}(\mathrm{Ct}=\mathrm{Ct}$ HOXD4$\mathrm{CtGAPDH})$. The smaller the $\mathrm{Ct}$ value predicted the higher expression of HOXD4.

\section{Immunohistochemical staining}

All paraffin sections proceed with $\mathrm{HE}$ staining and locating in the first place, $\mathrm{H}_{2} \mathrm{O}_{2}$ was used to eliminate endogenous activity of catalase and rinsed by PBS, subsequently sections were incubated with antibody (HOXD4) at $4^{\circ} \mathrm{C}$ overnight, we predicted that when the tan particles appeared, coloration was determined. IHC staining was estimated by two independent investigators. Results can be divided into four grades according to the dyeing intensity staining: 0 , negative; 1 , weak; 2 , moderate; and 3, strong, the percentage of immunostaining positive cells was also scored in four categories: $0(0$ $\%), 1$ (1-33 \%), 2 (34-66 \%), and 3 (67-100\%). The final staining score was defined to be the summation of positive staining intensity score and cell percentage score. Finally a HOXD4 expression score $\geq 4$ was regarded as high HOXD4 expression, meanwhile the score $\leq 3$ was low HOXD4 expression.

\section{Statistical analysis}

All data were tested for normality. The data of normal distribution were expressed as mean \pm standard deviation, and two samples were used for $t$ test. MannWhitney $U$ test was used to compare the mRNA expression level. Fisher's exact test was used to test possible associations between HOXD4 expression and clinicopathological feathers. Interval time from surgical treatment to the death or closest follow-up means overall survival (OS), meanwhile interval time from surgical treatment to the definite recurrence or closest follow-up means progression-free survival (PFS). Survival analysis curve was estimated using the Kaplan-Meier method, and appropriate variables were put into cox proportional hazards regression model to proceed multivariate analysis. When $p$ value was less than 0.05 , we admitted the statistical significance. GraphPad prism 5 (Graphpad Inc, La Jolla, USA) and IBM SPSS Statistics 23 (IBM Corp., Armonk, NY, USA) was used as data statistics software.

\section{Compliance with ethical standards}

\section{Informed consent}

Informed consent was obtained from all individual participants included in the study.

\section{Ethical approval}

All procedures performed in studies involving human participants were in accordance with the ethical standards of the institutional research committee of the First Affiliated Hospital of Zhengzhou University.

\section{ACKNOWLEDGMENTS}

The authors are thankful for the financial support from the Youth Innovation Fund of The First Affiliated Hospital of Zhengzhou University to Zhen-yu Zhang, the Youth Innovation Fund of The First Affiliated Hospital of Zhengzhou University to Yan-min Wang, the Youth 
Innovation Fund of The First Affiliated Hospital of Zhengzhou University to Ya-hui Bai, the Science and Technology Program of Henan Province to Jin-qiao Zhou (172102310648), and the Medical Science and Technology Program of Henan Province to Jin-qiao Zhou (201602058).

\section{CONFLICTS OF INTEREST}

Conflicts of interest relevant to this article was not reported.

\section{REFERENCES}

1. DeAngelis LM. Brain tumors. N Engl J Med. 2001; 344:114 23. https://doi.org/10.1056/nejm200101113440207.

2. Agnihotri S, Aldape KD, Zadeh G. Isocitrate dehydrogenase status and molecular subclasses of glioma and glioblastoma. Neurosurg Focus. 2014; 37:E13. https://doi.org/10.3171/20 14.9. focus 14505 .

3. Gao J, Ciriello G, Sander C, Schultz N. Collection, integration and analysis of cancer genomic profiles: from data to insight. Curr Opin Genet Dev. 2014; 24:92-8. https://doi.org/10.1016/j.gde.2013.12.003.

4. Young JS, Morshed RA, Kim JW, Balyasnikova IV, Ahmed AU, Lesniak MS. Advances in stem cells, induced pluripotent stem cells, and engineered cells: delivery vehicles for antiglioma therapy. Expert Opin Drug Deliv. 2014; 11:1733-46. https://doi.org/10.1517/17425247.2014.937420.

5. Stupp R, Mason WP, van den Bent MJ, Weller M, Fisher B, Taphoorn MJ, Belanger K, Brandes AA, Marosi C, Bogdahn U, Curschmann J, Janzer RC, Ludwin SK, et al. Radiotherapy plus concomitant and adjuvant temozolomide for glioblastoma. N Engl J Med. 2005; 352:987-96. https:// doi.org/10.1056/NEJMoa043330.

6. Armstrong TS, Wefel JS, Wang M, Gilbert MR, Won M, Bottomley A, Mendoza TR, Coens C, Werner-Wasik M, Brachman DG, Choucair AK, Mehta M. Net clinical benefit analysis of radiation therapy oncology group 0525: a phase III trial comparing conventional adjuvant temozolomide with dose-intensive temozolomide in patients with newly diagnosed glioblastoma. J Clin Oncol. 2013; 31:4076-84. https://doi.org/10.1200/jco.2013.49.6067.

7. Buckner JC. Factors influencing survival in high-grade gliomas. Semin Oncol. 2003; 30:10-4. https://doi. org/10.1053/j.seminoncol.2003.11.031.

8. Ricard D, Idbaih A, Ducray F, Lahutte M, HoangXuan K, Delattre JY. Primary brain tumours in adults. Lancet. 2012; 379:1984-96. https://doi.org/10.1016/ s0140-6736(11)61346-9.

9. Rich JN, Bigner DD. Development of novel targeted therapies in the treatment of malignant glioma. Nat Rev Drug Discov. 2004; 3:430-46. https://doi.org/10.1038/nrd1380.
10. Stewart LA. Chemotherapy in adult high-grade glioma: a systematic review and meta-analysis of individual patient data from 12 randomised trials. Lancet. 2002; 359:1011-8. https://doi.org/10.1016/ S0140-6736(02)08091-1.

11. Wen PY, Kesari S. Malignant gliomas in adults. N Engl J Med. 2008; 359:492-507. https://doi.org/10.1056/ NEJMra0708126.

12. Ampie L, Choy W, Lamano JB, Kesavabhotla K, Mao Q, Parsa AT, Bloch O. Prognostic factors for recurrence and complications in the surgical management of primary chordoid gliomas: a systematic review of literature. Clin Neurol Neurosurg. 2015; 138:129-36. https://doi. org/10.1016/j.clineuro.2015.08.011.

13. Lim YC, Roberts TL, Day BW, Stringer BW, Kozlov S, Fazry S, Bruce ZC, Ensbey KS, Walker DG, Boyd AW, Lavin MF. Increased sensitivity to ionizing radiation by targeting the homologous recombination pathway in glioma initiating cells. Mol Oncol. 2014; 8:1603-15. https://doi. org/10.1016/j.molonc.2014.06.012.

14. Laughon A. DNA binding specificity of homeodomains. Biochemistry. 1991; 30:11357-67.

15. Holland PW, Booth HA, Bruford EA. Classification and nomenclature of all human homeobox genes. BMC Biol. 2007; 5:47. https://doi.org/10.1186/1741-7007-5-47.

16. Botas J. Control of morphogenesis and differentiation by HOM/Hox genes. Curr Opin Cell Biol. 1993; 5:1015-22. https://doi.org/10.1016/0955-0674(93)90086-6.

17. Mark M, Rijli FM, Chambon P. Homeobox genes in embryogenesis and pathogenesis. Pediatr Res. 1997; 42:4219. https://doi.org/10.1203/00006450-199710000-00001.

18. Samuel S, Naora H. Homeobox gene expression in cancer: insights from developmental regulation and deregulation. Eur J Cancer. 2005; 41:2428-37. https://doi.org/10.1016/j. ejca.2005.08.014.

19. Ross SA, McCaffery PJ, Drager UC, De Luca LM. Retinoids in embryonal development. Physiol Rev. 2000; 80:1021-54. 1021-1054, 2000.

20. DeInnocentes P, Perry AL, Graff EC, Lutful Kabir FM, Curtis Bird R. Characterization of HOX gene expression in canine mammary tumour cell lines from spontaneous tumours. Vet Comp Oncol. 2015; 13:322-36. https://doi. org/10.1111/vco.12062.

21. Zha Y, Ding E, Yang L, Mao L, Wang X, McCarthy BA, Huang S, Ding HF. Functional dissection of HOXD cluster genes in regulation of neuroblastoma cell proliferation and differentiation. PLoS One. 2012; 7:e40728. https://doi. org/10.1371/journal.pone.0040728.

22. Feiden S, Feiden W. [WHO classification of tumours of the CNS: revised edition of 2007 with critical comments on the typing und grading of common-type diffuse gliomas]. [Article in German]. Pathologe. 2008; 29:411-21. https:// doi.org/10.1007/s00292-008-1064-5. 
23. Kmita M, Duboule D. Organizing axes in time and space; 25 years of colinear tinkering. Science. 2003; 301:331-3. https://doi.org/10.1126/science.1085753.

24. Quentmeier H, Dirks WG, Macleod RA, Reinhardt J, Zaborski M, Drexler HG. Expression of HOX genes in acute leukemia cell lines with and without MLL translocations. Leuk Lymphoma. 2004; 45:567-74. https://doi.org/10.108 0/10428190310001609942.

25. Care A, Felicetti F, Meccia E, Bottero L, Parenza M, Stoppacciaro A, Peschle C, Colombo MP. HOXB7: a key factor for tumor-associated angiogenic switch. Cancer Res. $2001 ; 61: 6532-9$.

26. Costa BM, Smith JS, Chen Y, Chen J, Phillips HS, Aldape KD, Zardo G, Nigro J, James CD, Fridlyand J, Reis RM, Costello JF. Reversing HOXA9 oncogene activation by PI3K inhibition: epigenetic mechanism and prognostic significance in human glioblastoma. Cancer Res. 2010; 70:453-62. https://doi.org/10.1158/0008-5472.can-09-2189.

27. Duan R, Han L, Wang Q, Wei J, Chen L, Zhang J, Kang C, Wang L. HOXA13 is a potential GBM diagnostic marker and promotes glioma invasion by activating the Wnt and TGF-beta pathways. Oncotarget. 2015; 6:27778-93. https:// doi.org/10.18632/oncotarget.4813.

28. Buccoliero AM, Castiglione F, Rossi Degl'Innocenti D, Ammanati F, Giordano F, Sanzo M, Mussa F, Genitori L, Taddei GL. Hox-D genes expression in pediatric low-grade gliomas: real-time-PCR study. Cell Mol Neurobiol. 2009; 29:1-6. https://doi.org/10.1007/s10571-008-9282-1.

29. Tabuse M, Ohta S, Ohashi Y, Fukaya R, Misawa A, Yoshida K, Kawase T, Saya H, Thirant C, Chneiweiss H, Matsuzaki Y, Okano H, Kawakami Y, et al. Functional analysis of HOXD9 in human gliomas and glioma cancer stem cells. Mol Cancer. 2011; 10:60. https://doi. org/10.1186/1476-4598-10-60.
30. Jansen M, Yip S, Louis DN. Molecular pathology in adult gliomas: diagnostic, prognostic, and predictive markers. Lancet Neurol. 2010; 9:717-26. https://doi.org/10.1016/ s1474-4422(10)70105-8.

31. Filippini G, Falcone C, Boiardi A, Broggi G, Bruzzone MG, Caldiroli D, Farina R, Farinotti M, Fariselli L, Finocchiaro G, Giombini S, Pollo B, Savoiardo M, et al. Prognostic factors for survival in 676 consecutive patients with newly diagnosed primary glioblastoma. Neuro Oncol. 2008; 10:79-87. https://doi.org/10.1215/15228517-2007-038.

32. Barker FG, Prados MD, Chang SM, Davis RL, Gutin PH, Lamborn KR, Larson DA, McDermott MW, Sneed $\mathrm{PK}$, Wilson CB. Bromodeoxyuridine labeling index in glioblastoma multiforme: relation to radiation response, age, and survival. Int J Radiat Oncol Biol Phys. 1996; 34:803-8. https://doi.org/10.1016/0360-3016(95)02027-6.

33. Lv S, Dai C, Liu Y, Shi R, Tang Z, Han M, Bian R, Sun B, Wang R. Retraction note to: the impact of survivin on prognosis and clinicopathology of glioma patients: a systematic meta-analysis. Mol Neurobiol. 2017. https://doi. org/10.1007/s12035-017-0402-0.

34. van den Bent MJ. Interobserver variation of the histopathological diagnosis in clinical trials on glioma: a clinician's perspective. Acta Neuropathol. 2010; 120:297304. https://doi.org/10.1007/s00401-010-0725-7.

35. Eckel-Passow JE, Lachance DH, Molinaro AM, Walsh KM, Decker PA, Sicotte H, Pekmezci M, Rice T, Kosel ML, Smirnov IV, Sarkar G, Caron AA, Kollmeyer TM, et al. Glioma groups based on 1p/19q, IDH, and TERT promoter mutations in tumors. N Engl J Med. 2015; 372:2499-508. https://doi.org/10.1056/NEJMoa1407279.

36. Ohgaki $\mathrm{H}$, Kleihues $\mathrm{P}$. The definition of primary and secondary glioblastoma. Clin Cancer Res. 2013; 19:764-72. https://doi.org/10.1158/1078-0432.ccr-12-3002. 